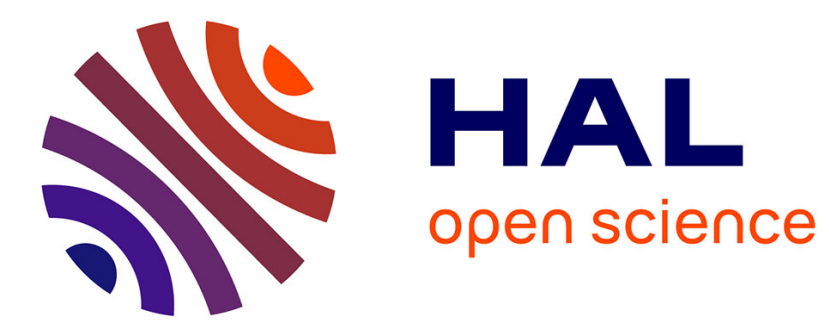

\title{
Backstepping boundary observer based-control for hyperbolic PDE in rotary drilling system
}

Rhouma Mlayeh, Samir Toumi, Lotfi Beji

\section{To cite this version:}

Rhouma Mlayeh, Samir Toumi, Lotfi Beji. Backstepping boundary observer based-control for hyperbolic PDE in rotary drilling system. Applied Mathematics and Computation, 2018, 322, pp.66-78. 10.1016/j.amc.2017.11.034 . hal-01674970

\section{HAL Id: hal-01674970 \\ https://hal.science/hal-01674970}

Submitted on 11 Nov 2019

HAL is a multi-disciplinary open access archive for the deposit and dissemination of scientific research documents, whether they are published or not. The documents may come from teaching and research institutions in France or abroad, or from public or private research centers.
L'archive ouverte pluridisciplinaire HAL, est destinée au dépôt et à la diffusion de documents scientifiques de niveau recherche, publiés ou non, émanant des établissements d'enseignement et de recherche français ou étrangers, des laboratoires publics ou privés. 


\title{
Backstepping boundary observer based-control for hyperbolic PDE in rotary drilling system
}

\author{
Rhouma Mlayeh ${ }^{\mathrm{b}, *}$, Samir Toumi ${ }^{\mathrm{a}, \mathrm{b}}$, Lotfi Beji ${ }^{\mathrm{a}}$ \\ a IBISC-EA 4526 laboratory, University of Evry, 40 rue du Pelvoux, Evry 91020, France \\ ${ }^{\mathrm{b}}$ LIM laboratory, Polytechnic School of Tunisia, BP 743, La Marsa 2078, Tunisia
}

\begin{abstract}
It is well known that torsional vibrations in oil well system affect the drilling directions and may be inherent for drilling systems. The drill pipe model is described by second order hyperbolic Partial Differential Equation (PDE) with mixed boundary conditions in which a sliding velocity is considered at the top end. In this paper, we consider the problem of boundary observer design for one-dimensional PDE with the usually neglected damping term. The main purpose is the construction of a control law which stabilizes the damped wave PDE, using only boundary measurements. From the Lyapunov theory, we show an exponentially vibration stability of the partially equipped oil well drilling system. The observer-based control law is found using the backstepping approach for second-order hyperbolic PDE. The numerical simulations confirm the effectiveness of the proposed PDE observer based controller.
\end{abstract}

\section{Introduction}

A common type of instability in oil well drilling system is stick-slip oscillation (more details in [1]), caused by friction between the drill bit and the rock resulting in torsional vibrations of the drill string, which reduce penetration rates and increase drilling operation costs. The stick-slip phenomenon is an undesirable limit cycle of the drill string velocity yielding potentially significant damages on oil production facilities. In the last century, many research effort on the avoiding torsional vibrations has been proposed [2-10]. Despite the development of several techniques for eliminating torsional vibrations (stick-slip oscillations), nowadays many problems remains open for drilling systems. The torsional dynamics of a drill string are modeled as a damped wave PDE that governs the dynamics of the angular displacement of the drill string. Based on the linearization of its dynamics, a control method for the stabilization of the drilling instability is presented in [11]. The energy function is proposed by Saldivar et al. in [6] for the torsional distributed model allows to find a control law that ensures the energy dissipation during the drilling.

In [12] the authors are developed a simplified model, where there is no damping in the domain and the drill bit has no inertia and have proposed an output feedback adaptive controller. The anti-damping wave equation used in the paper by Bresch-Pietri and Krstic [12] is only an approximation of the model commonly used in our paper to account for the stick-slip phenomenon in which a friction ODE is used as the boundary condition instead.

\footnotetext{
* Corresponding author.

E-mail address: rhouma.mlayeh@ipeit.rnu.tn (R. Mlayeh).
} 


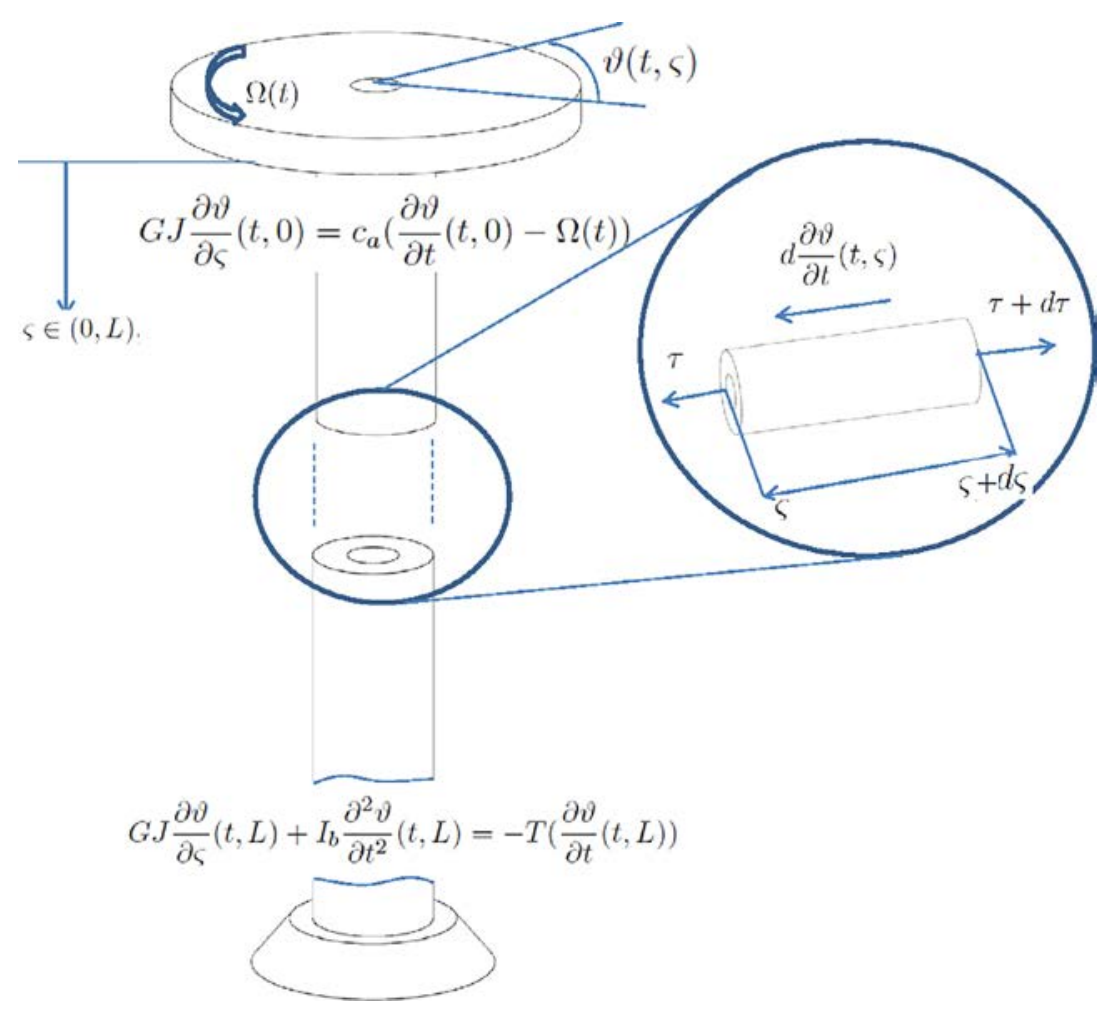

Fig. 1. Drilling system.

In this work, we are concerned with the problem of boundary observer stabilization for a system of hyperbolic PDE which describes the drilling systems. Basically, in our designs we use the backstepping techniques (more details in [13]) and the Lyapunov theory to study the stability analysis. Initially, the backstepping approach developed for parabolic equations, it has been applied to nonlinear PDE, first-order hyperbolic equations, second-order hyperbolic equations, fluid flow $[13,14]$. Historically, in 1990, the backstepping approach is well known in ordinary differential equations (ODE) stability. It is developed by Kokotovic [15] for analyzing the stability of nonlinear ordinary differential equation. It has the ability to cope with the control synthesis, and around 2000 this technique becomes a useful tool in the boundary control of PDE [13]. The main purposes of this work are: first, the design of an observer using only boundary velocity measurements at the top and the construction of an observation error system; second, the development of a control law taking into account in-domain damping usually neglected; and finally, the well-posedness problem of the observer torsional vibration. We use the backstepping approach to design a full-state feedback observer law that makes the closed-loop system exponentially stable. The stability analysis is conducted with infinite-dimensional backstepping transformations for the damped wave PDE state and by constructing a Lyapunov functional.

The paper is structured as follows. In Section 2, we recall the PDE with the boundary conditions that permits to describe the torsional vibration problem. An observer based control law is presented in this section. In Section 3, we find the output injection gain and we prove the convergence of the estimation error system using Lyapunov theory and backstepping technique. The simulation results are given in Section 4. Some concluding remarks and perspectives are also introduced.

\section{Boundary observer based control}

\subsection{Distributed parameter model: damped wave equation}

A more exhaustive description of the rotary system can be found in [1]. One of the principal problems is the appearance of oscillatory behaviors, that cause a decreasing of the drilling performance from the view points of different parameters (rotational speed of the bit, rate of penetration at the surface) and so provoking the mechanical failure of the drill string. Some causes of stick-slip oscillations are backlash between contacting parts, nonlinear damping, hysteresis, and geometrical imperfections which are very difficult to model. However, the main cause of such vibrations in drill string is the friction appearing by contact with the rock formation [16]. Accordingly, a model describing the drill string behavior should include a bit-rock friction torque model adequate enough to properly reproduce this effect (Fig. 1). 
The dynamic of the torsional variable $\vartheta(t, \varsigma)$ along the drill pipe is governed by $[4,6,17]$ :

$$
G J \vartheta_{\varsigma \varsigma}(t, \varsigma)-I \vartheta_{t t}(t, \varsigma)-\sigma \vartheta_{t}(t, \varsigma)=0
$$

$\varsigma \in(0, L), t \in(0,+\infty)$, with the boundary conditions

$$
\begin{aligned}
& G J \vartheta_{\varsigma}(t, 0)=c_{a}\left(\vartheta_{t}(t, 0)-\omega(t)\right) \\
& G J \vartheta_{\varsigma}(t, L)+I_{b} \vartheta_{t t}(t, L)=-T\left(\vartheta_{t}(t, L)\right)
\end{aligned}
$$

where $L$ is the length of the drill pipe, $I$ is the inertia, $G$ the shear modulus, $I_{b}$ is chosen to represent the assembly at the bottom hole, $J$ the geometrical moment of inertia, $c_{a}$ the sliding torque coefficient, $\sigma$ the drill string damping, and $\omega$ the control input (angular velocity due to the rotary table). The extremity $(\zeta=L)$, is subject to a torque on the bit $T\left(\frac{\partial \vartheta}{\partial t}(t, L)\right)$, which is a function of the bit velocity [18].

In order to improve clarity, we introduce the normalized rod length $x=\frac{S}{L}$, and the next variable change [2]:

$$
v(t, x)=\vartheta\left(L \sqrt{\frac{I}{G J}} t, L(1-x)\right), x \in(0,1) .
$$

Then, the dynamic of the torsional variable reads

$$
\begin{aligned}
& v_{t t}(t, x)=v_{x x}(t, x)-\iota v_{t}(t, x) \\
& v_{x}(t, 1)=\Omega(t) \\
& v_{t t}(t, 0)=a v_{x}(t, 0)+a F\left(v_{t}(t, 0)\right)
\end{aligned}
$$

where

$$
\Omega(t)=\frac{c_{a} L}{G J}\left(\omega(t)-\frac{1}{L} \sqrt{\frac{G J}{I}} v_{t}(t, 1)\right), \iota=\sigma L \sqrt{\frac{1}{I G]}}, F\left(v_{t}(t, 0)\right)=-\frac{L}{G J} T\left(\frac{1}{L} \sqrt{\frac{G J}{I}} v_{t}(t, 0)\right) \text {, and } a=\frac{L I}{I_{b}} .
$$

To linearize the tip boundary condition (7), we use the next form [3]

$$
\bar{v}(t, x)=\frac{\iota w_{r}}{2} x^{2}-F\left(w_{r}\right) x+w_{r} t+v_{0}
$$

as a reference trajectory, such that $w_{r}=\bar{v}_{t}(t, x)$.

Then we obtain the next linearized of equations system

$$
\begin{aligned}
& v_{t t}(t, x)=v_{x x}(t, x)-\iota v_{t}(t, x) \\
& v_{x}(t, 1)=\Omega(t) \\
& v_{t t}(t, 0)=a v_{x}(t, 0)+a b v_{t}(t, 0)
\end{aligned}
$$

where $b=\frac{\partial F\left(w_{r}\right)}{\partial u}$ and $u(t)=v_{t}(t, 1)$.

One of the main challenge during drilling operation lies in the poor knowledge of the downhole conditions (pressure and temperature conditions, gas and oil ratios). In the next, we propose an approach to estimate unknown parameters while drilling oil well. Hence, the main purpose in this study, is the stability analysis of the observer PDE which encountered in an drilling system.

In this section, we design an observer for the system given above when one boundary measurement is available. We assume that velocity at $x=1$ is measured (i.e. the top boundary condition, meaning the drill string head).

We denote the estimates by a widehat, and we construct system behavior that integrates from an output injection term:

$$
\begin{aligned}
& \widehat{v}_{t t}(t, x)=\widehat{v}_{x x}(t, x)-\imath \widehat{v}_{t}(t, x) \\
& \widehat{v}_{x}(t, 1)=\Omega(t)-\gamma(v(t, 1)-\widehat{v}(t, 1)) \\
& \widehat{v}_{t t}(t, 0)=a \widehat{v}_{x}(t, 0)+a b \widehat{v}_{t}(t, 0)
\end{aligned}
$$

where $\gamma$ is the output injection gain to be designed. 


\subsection{Observer target system and backstepping transformation}

This section shows the importance of observer target system, backstepping techniques, and the Lyapunov theory, providing a useful analysis for stability in oil well drilling system. Here, the main purpose is to find a control law $\Omega(t)$ that transforms (12)-(14) to a next designed observer target system,

$$
\begin{aligned}
& \widehat{w}_{t t}(t, x)=\widehat{w}_{x x}(t, x)-\iota \widehat{w}_{t}(t, x) \\
& \widehat{w}_{x}(t, 1)=0 \\
& \widehat{w}_{t t}(t, 0)=a e^{-\eta} \widehat{w}_{x}(t, 0)-(2 a \epsilon+1) \widehat{w}_{t}(t, 0) .
\end{aligned}
$$

The $\eta$ and $\epsilon$ parameters will be defined by the following Lemma.

Lemma 1. Let us introduce the function

$$
V(t)=\frac{1}{2}\left[\int_{0}^{1}\left(e^{-\eta}\left(\widehat{w}_{x}\right)^{2}+e^{-\eta}\left(\widehat{w}_{t}\right)^{2}+\epsilon e^{-\eta x}(1-x) \widehat{w}_{t} \widehat{w}_{x}\right) d x\right]+\frac{1}{a}\left(\widehat{w}_{t}(t, 0)\right)^{2},
$$

with $\frac{1}{2}>\epsilon>0, \eta \leq-\frac{2+\epsilon}{\epsilon(1-x)}$, such that $x \in[0,1[$, and the norm $\widehat{\Xi}$ where

$$
\widehat{\Xi}^{2}(t)=\left\|\widehat{w}_{t}\right\|_{L^{2}([0,1])}^{2}+\left\|\widehat{w}_{x}\right\|_{L^{2}([0,1])}^{2}+\left|\widehat{w}_{t}(t, 0)\right|^{2} .
$$

Then $n_{1} \widehat{\Xi}^{2}(t) \leq V(t) \leq n_{2} \widehat{\Xi}^{2}(t)$ where $n_{1}=\min \left\{\frac{e^{-\eta}}{2}-\frac{\epsilon e^{-\eta}}{4}, \frac{1}{a}\right\}$ and $n_{2}=\max \left\{\frac{e^{-\eta}}{2}+\frac{\epsilon e^{-\eta}}{4}, \frac{1}{a}\right\}$.

Proof. Using the Cauchy-Schwarz and Young's inequalities, we obtain

$$
\begin{aligned}
V(t) & =\frac{1}{2}\left[\int_{0}^{1}\left(e^{-\eta}\left(\widehat{w}_{x}\right)^{2}+e^{-\eta}\left(\widehat{w}_{t}\right)^{2}+\epsilon e^{-\eta x}(1-x) \widehat{w}_{t} \widehat{w}_{x}\right) d x\right]+\frac{1}{a}\left(\widehat{w}_{t}(t, 0)\right)^{2} \\
& \geq \frac{e^{-\eta}}{2}\left\|\widehat{w}_{t}\right\|^{2}+\frac{e^{-\eta}}{2}\left\|\widehat{w}_{x}\right\|^{2}+\frac{1}{a}\left|\widehat{w}_{t}(t, 0)\right|^{2}-\frac{\epsilon e^{-\eta}}{2} \int_{0}^{1}\left|\widehat{w}_{t} w_{x}\right| d x \\
& \geq\left(\frac{e^{-\eta}}{2}-\frac{\epsilon e^{-\eta}}{4}\right)\left(\left\|\widehat{w}_{t}\right\|^{2}+\left\|\widehat{w}_{x}\right\|^{2}\right)+\frac{1}{a}\left|\widehat{w}_{t}(t, 0)\right|^{2} \\
& \geq \min \left\{\frac{e^{-\eta}}{2}-\frac{\epsilon e^{-\eta}}{4}, \frac{1}{a}\right\} \widehat{\Xi}^{2}(t) .
\end{aligned}
$$

On the other hand,

$$
\begin{aligned}
V(t) & =\frac{1}{2}\left[\int_{0}^{1}\left(e^{-\eta}\left(\widehat{w}_{x}\right)^{2}+e^{-\eta}\left(\widehat{w}_{t}\right)^{2}+\epsilon e^{-\eta x}(1-x) \widehat{w}_{t} \widehat{w}_{x}\right) d x+\frac{1}{a}\left(\widehat{w}_{t}(t, 0)\right)^{2}\right] \\
& \leq \frac{e^{-\eta}}{2}\left\|\widehat{w}_{t}\right\|^{2}+\frac{e^{-\eta}}{2}\left\|\widehat{w}_{x}\right\|^{2}+\frac{1}{a}\left|w_{t}(t, 0)\right|^{2}+\frac{\epsilon e^{-\eta}}{2} \int_{0}^{1}\left|\tilde{w}_{t} \tilde{w}_{x}\right| d x \\
& \leq\left(\frac{e^{-\eta}}{2}+\frac{\epsilon e^{-\eta}}{4}\right)\left(\left\|\widehat{w}_{t}\right\|^{2}+\left\|\widehat{w}_{x}\right\|^{2}\right)+\frac{1}{a}\left|w_{t}(t, 0)\right|^{2} \\
& \leq \max \left\{\frac{e^{-\eta}}{2}+\frac{\epsilon e^{-\eta}}{4}, \frac{1}{a}\right\} \widehat{\Xi}^{2}(t) .
\end{aligned}
$$

Then $n_{1} \widehat{\Xi}^{2}(t) \leq V(t) \leq n_{2} \widehat{\Xi}^{2}(t)$ with $n_{1}=\min \left\{\frac{e^{-\eta}}{2}-\frac{\epsilon e^{-\eta}}{4}, \frac{1}{a}\right\}$ and $n_{2}=\max \left\{\frac{e^{-\eta}}{2}+\frac{\epsilon e^{-\eta}}{4}, \frac{1}{a}\right\}$.

Now, we are interested in the stabilization of the observer target system.

Theorem 1. (Observer target system stability) Consider system (15)-(17), with initial condition $\widehat{w}_{0}=\widehat{w}(0, x) \in L^{2}([0,1])$. Then the zero equilibrium of the system (15)-(17) is exponentially stable in the sense of the next norm

$$
\widehat{\Xi}^{2}(t)=\left\|\widehat{w}_{t}\right\|_{L^{2}([0,1])}^{2}+\left\|\widehat{w}_{x}\right\|_{L^{2}([0,1])}^{2}+\left|\widehat{w}_{t}(t, 0)\right|^{2} .
$$

Proof. In order to prove the observer target system stability, let us consider the proposed $V(t)$ as a Lyapunov function candidate,

$$
V(t)=\frac{1}{2}\left[\int_{0}^{1}\left(e^{-\eta}\left(\widehat{w}_{x}\right)^{2}+e^{-\eta}\left(\widehat{w}_{t}\right)^{2}+\epsilon e^{-\eta x}(1-x) \widehat{w}_{t} \widehat{w}_{x}\right) d x\right]+\frac{1}{a}\left(\widehat{w}_{t}(t, 0)\right)^{2}
$$


Differentiating $V$ with respect to time, we get

$$
\begin{aligned}
\dot{V}(t)= & \int_{0}^{1}\left(e^{-\eta} \widehat{w}_{t x} \widehat{w}_{x}+e^{-\eta} \widehat{w}_{t t} \widehat{w}_{t}+\frac{1}{2} \epsilon e^{-\eta x}(1-x) \widehat{w}_{t t} \widehat{w}_{x}+\frac{1}{2} \epsilon e^{-\eta x}(1-x) \widehat{w}_{t x} \widehat{w}_{t}\right) d x \\
& +\frac{1}{a} \widehat{w}_{t t}(t, 0) \widehat{w}_{t}(t, 0) \\
= & -\iota e^{-\eta} \int_{0}^{1} \widehat{w}_{t}^{2}+\int_{0}^{1}\left(e^{-\eta} \widehat{w}_{t x} \widehat{w}_{x}+e^{-\eta} \widehat{w}_{x x} \widehat{w}_{t}+\frac{1}{2} \epsilon e^{-\eta x}(1-x) \widehat{w}_{x x} \widehat{w}_{x}\right. \\
& \left.+\frac{1}{2} \epsilon(1-x) \widehat{w}_{t x} \widehat{w}_{t}\right) d x+\frac{1}{a}\left(\widehat{w}_{t t}(t, 0) \widehat{w}_{t}(t, 0)\right)-\frac{\iota \epsilon}{2} \int_{0}^{1} e^{-\eta x}(1-x) \widehat{w}_{t} \widehat{w}_{x} d x \\
= & -e^{-\eta} \widehat{w}_{t}(t, 0) \widehat{w}_{x}(t, 0)-\frac{\epsilon}{4} \widehat{w}_{x}(t, 0)^{2}-\frac{\epsilon}{4} \widehat{w}_{t}(t, 0)^{2}-\int_{0}^{1} \frac{-\epsilon-\eta \epsilon(1-x)}{2} e^{-\eta x} \frac{\widehat{w}_{x}^{2}}{2} d x \\
& -\frac{2 a \epsilon+1}{a} \widehat{w}_{t}^{2}(t, 0)+e^{-\eta} \widehat{w}_{t}(t, 0) \widehat{w}_{x}(t, 0) \\
& -\int_{0}^{1} \frac{-\epsilon-\eta \epsilon(1-x)}{2} e^{-\eta x} \frac{\widehat{w}_{t}^{2}}{2} d x-\frac{\iota}{2} \int_{0}^{1} \epsilon e^{-\eta x}(1-x) \widehat{w}_{t} \widehat{w}_{x} d x-\iota \int_{0}^{1} e^{-\eta} \widehat{w}_{t}^{2} d x \\
\leq & -e^{\eta} \int_{0}^{1} e^{-\eta} \frac{\widehat{w}_{x}^{2}}{2} d x-\frac{1}{a} \widehat{w}_{t}^{2}(t, 0)-\frac{\iota}{2} \int_{0}^{1} \epsilon e^{-\eta x}(1-x) \widehat{w}_{t} \widehat{w}_{x} d x-\iota \int_{0}^{1} e^{-\eta} \widehat{w}_{t}^{2} d x \\
\leq & -\min \left(e^{\eta}, \iota, 1\right) V(t) .
\end{aligned}
$$

By Lemma 1 , we have

$$
n_{1} \widehat{\Xi}^{2}(t) \leq V(t) \leq n_{2} \widehat{\Xi}^{2}(t) .
$$

Hence there exist $c>0$ and $k \geq 0$ such that

$$
\widehat{\Xi}(t) \leq c e^{-k t} \widehat{\Xi}(0) \text {. }
$$

This implies that the observer target system (15)-(17) is exponentially stable at the equilibrium in the sense of the $\widehat{\Xi}$ norm.

In order to convert the observer plant into the observer target systems (i.e. $\widehat{v}(t, x) \longrightarrow \widehat{w}(t, x)$ ), we consider the next backstepping transformation

$$
\begin{aligned}
\widehat{w}(t, x)= & \widehat{v}(t, x)-\int_{0}^{x} k(x, \xi) \widehat{v}(t, \xi) d \xi-\beta(x) \widehat{v}(t, 0)-\int_{0}^{x} p(x, \xi) \widehat{v}_{t}(t, \xi) d \xi \\
& -\int_{0}^{x} l(x, \xi) \widehat{v}_{\xi}(t, \xi) d \xi .
\end{aligned}
$$

Plugging the backstepping transformation (18) into the observer target system (15)-(17), integrating by parts, and using the boundary conditions, we obtain:

- Kernel surface terms $(x, \xi)$ :

$$
\begin{aligned}
& l_{\xi \xi}(x, \xi)=l_{x x}(x, \xi), \\
& k_{\xi \xi}(x, \xi)=k_{x x}(x, \xi), \\
& p_{\xi \xi}(x, \xi)=p_{x x}(x, \xi),
\end{aligned}
$$

- Kernel diagonal terms $(x, x)$ :

$$
l_{x}(x, x)=0, \quad k_{x}(x, x)=0, \quad p_{x}(x, x)=0 .
$$

- Kernel vertical terms $(x, 0)$ and point-wise terms $(0,0)$ :

$$
\begin{aligned}
& l_{\xi}(x, 0)=k(x, 0), \quad k_{\xi}(x, 0)=\beta^{\prime \prime}(x), \\
& p(x, 0)=0, \quad p_{\xi}(x, 0)=0 \\
& l(x, 0)=\beta(x), l(0,0)=\beta(0) \\
& k_{\xi}(0,0)=a e^{-\eta} k(0,0)+a e^{-\eta} \beta^{\prime}(0)=\beta^{\prime \prime}(0) .
\end{aligned}
$$


The kernel of the backstepping transformation satisfies an interesting system of wave PDE which is easily solvable. These equations are defined on a triangular domain $\Delta=\left\{(x, \xi) \in \mathbb{R}^{2}: 0 \leq \xi \leq x \leq 1\right\}$.

At this step, introducing the backstepping transformation (18) into (16), we deduce the next control law

$$
\begin{aligned}
\Omega(t)= & \frac{1}{1-l(1,1)}\left[k(1,1) \widehat{v}(t, 1)+\int_{0}^{1} k_{x}(1, \xi) \widehat{v}(t, \xi) d \xi+p(1,1) \widehat{v}_{t}(t, 1)\right. \\
& \left.+\int_{0}^{1} p_{x}(1, \xi) \widehat{v}_{t}(t, \xi) d \xi+\int_{0}^{1} l_{x}(1, \xi) \widehat{v}_{\xi}(t, \xi) d \xi+\beta^{\prime}(1) \widehat{v}(t, 0)\right] \\
& +\gamma(v(t, 1)-\widehat{v}(t, 1)) .
\end{aligned}
$$

It is worth noticing that $1-l(1,1)=1-\beta(0) \neq 0$ and cannot be zero since $\beta(0) \neq 1$ here.

It remains to study the behavior of the observer plant system from the inverse backstepping transformation (i.e. $\widehat{w}(t, x) \rightarrow \widehat{v}(t, x))$ and the stability conditions under the control law (27). Let us consider the inverse backstepping transformation as follows

$$
\begin{aligned}
\widehat{v}(t, x) & =\widehat{w}(t, x)+\int_{0}^{x} e(x, \xi) \widehat{w}(t, \xi) d \xi+\int_{0}^{x} f(x, \xi) \widehat{w}_{t}(t, \xi) d \xi+\pi(x) \widehat{w}(t, 0) \\
& +\int_{0}^{x} h(x, \xi) \widehat{w}_{\xi}(t, \xi) d \xi .
\end{aligned}
$$

Introducing the expression (28) into the observer plant system (12)-(14), we find:

- Kernel surface terms $(x, \xi)$ :

$$
\begin{aligned}
& h_{\xi \xi}(x, \xi)=h_{x x}(x, \xi), \\
& e_{\xi \xi}(x, \xi)=e_{x x}(x, \xi), \\
& f_{\xi \xi}(x, \xi)=f_{x x}(x, \xi),
\end{aligned}
$$

- Kernel diagonal terms $(x, x)$ :

$$
h_{x}(x, x)=0, \quad e_{x}(x, x)=0, \quad f_{x}(x, x)=0 .
$$

- Kernel vertical terms $(x, 0)$ and point-wise terms $(0,0)$ :

$$
\begin{aligned}
& e(x, 0)=h_{\xi}(x, 0), \quad e_{\xi}(x, 0)=\pi^{\prime \prime}(x), \quad \pi^{\prime}(0)=0 \\
& f(x, 0)=0, \quad h(x, 0)=\pi(x), \quad f_{\xi}(x, 0)=0 \\
& e(0,0)=h_{\xi}(0,0)=0, \quad h(0,0)=\pi(0)=-1 .
\end{aligned}
$$

It is easily to verify that this equations are defined on a triangular domain $\Delta=\left\{(x, \xi) \in \mathbb{R}^{2}: 0 \leq \xi \leq x \leq 1\right\}$.

The main result regarding the observer plant system stability is summarized in the next Theorem.

Theorem 2. (Observer plant system stability) Consider system (12)-(14) with initial condition $v_{0} \in L^{2}([0,1])$, and with control law (27) where the kernels $k, p$, and $l$ are obtained from (19)-(26). Then the system (12)-(14) is exponentially stable at the zero equilibrium in the sense of the next norm

$$
\widehat{\Pi}^{2}(t)=\|\widehat{v}(t, .)\|_{L^{2}([0,1])}^{2}+\left\|\widehat{v}_{t}(t, .)\right\|_{L^{2}([0,1])}^{2}+\left\|\widehat{v}_{x}(t, .)\right\|_{L^{2}([0,1])}^{2}+\left|\widehat{v}_{t}(t, 0)\right|^{2} .
$$

Proof. Firstly, we denote by $L^{2}=L^{2}([0,1])$ and let introduce the next norms (for example) as: $\beta_{\infty}=\sup _{x \in[0,1]}|\beta(x)|, k_{\infty}=$ $\max _{(x, \xi) \in \Delta}\||k(x, \xi)|\|_{2}^{2}$, and so on for $l_{\infty},\left(p_{\xi \xi}\right)_{\infty}, p_{\infty}$, where $\||k(x, \xi)|\|_{2}^{2}$ denotes the classical operator norm. We will prove that there exist $\zeta_{1}>0$ and $\zeta_{2}>0$ such that

$$
\zeta_{1} \widehat{\Xi}(t) \leq \widehat{\Pi}(t) \leq \zeta_{2} \widehat{\Xi}(t)
$$

Recall that $p_{\xi}(x, 0)=0, p(x, 0)=0, p_{x}(x, x)=0, l(x, 0)=\beta(x)$. Consequently, $\widehat{w}_{t}$ is rewritten in this form

$$
\begin{aligned}
\widehat{w}_{t}(t, x)= & \widehat{v}_{t}(t, x)-\int_{0}^{x} k(x, \xi) \widehat{v}_{t}(t, \xi) d \xi-p(x, x) \widehat{v}_{x}(t, x)-\int_{0}^{x} p_{\xi \xi}(x, \xi) \widehat{v}(t, \xi) d \xi \\
& +\int_{0}^{x} \iota p(x, \xi) \widehat{v}_{t}(t, \xi) d \xi-l(x, x) \widehat{v}_{t}(t, x)+\int_{0}^{x} l_{\xi}(x, \xi) \widehat{v}_{t}(t, \xi) d \xi \\
& -\beta(x) \widehat{v}_{t}(t, 0) .
\end{aligned}
$$

Using Cauchy-Schwarz's inequalities, we prove

$$
\begin{aligned}
\left\|\widehat{w}_{t}(t, .)\right\|_{L^{2}}^{2} \leq & \left(1+k_{\infty}+l_{\infty}+\left(l_{\xi}\right)_{\infty}+\iota p_{\infty}\right)\left\|\widehat{v}_{t}(t, .)\right\|_{L^{2}}^{2}+p_{\infty}\left\|\widehat{v}_{x}(t, .)\right\|_{L^{2}}^{2} \\
& +\left(\left(p_{\xi \xi}\right)_{\infty}\|\widehat{v}(t, .)\|_{L^{2}}^{2}+\beta_{\infty}\left|\widehat{v}_{t}(t, 0)\right|^{2}\right) \\
\leq & a_{1} \widehat{\Pi}^{2}
\end{aligned}
$$


where $a_{1}=\max \left\{1+k_{\infty}+l_{\infty}+\left(l_{\xi}\right)_{\infty}+\iota p_{\infty},\left(p_{\xi \xi}\right)_{\infty}, p_{\infty}, \beta_{\infty}\right\}$.

As $\widehat{v}(t, 0)=\widehat{v}(t, x)-\int_{0}^{x} \widehat{v}_{y}(t, y) d y$, we find

$$
\left\|\widehat{w}_{x}(t, .)\right\|_{L^{2}}^{2} \leq a_{2}\left(\left\|\widehat{v}_{x}(t, .)\right\|_{L^{2}}^{2}+\|\widehat{v}(t, .)\|_{L^{2}}^{2}+\left\|\widehat{v}_{t}(t, .)\right\|_{L^{2}}^{2}\right)
$$

where $a_{2}=\max \left\{1+l_{\infty}+\left(l_{x}\right)_{\infty}+\beta_{\infty}^{\prime}, k_{\infty}+\left(k_{x}\right)_{\infty}+\beta_{\infty}^{\prime}, p_{\infty}+\left(p_{x}\right)_{\infty}\right\}$.

Also, we have $\left|\widehat{w}_{t}(t, 0)\right|^{2} \leq \mid \widehat{v}_{t}\left(t,\left.0\right|^{2}\right.$. Hence, there exist $\zeta_{1}>0$ such that $\zeta_{1} \widehat{\Xi}(t) \leq \widehat{\Pi}(t)$.

Recall that the inverse backstepping transformation is given by

$$
\begin{aligned}
\widehat{v}(t, x)= & \widehat{w}(t, x)+\int_{0}^{x} e(x, \xi) \widehat{w}(t, \xi) d \xi+\pi(x) \widehat{w}(t, 0) \\
& +\int_{0}^{x} f(x, \xi) \widehat{w}_{t}(t, \xi) d \xi+\int_{0}^{x} h(x, \xi) \widehat{w}_{\xi}(t, \xi) d \xi .
\end{aligned}
$$

As $\widehat{w}(t, 0)=\widehat{w}(t, x)-\int_{0}^{x} \widehat{w}_{y}(t, y) d y$, using Poincare's inequality, we obtain

$$
\|\widehat{v}(t, .)\|_{L^{2}}^{2} \leq a_{3}\left(\left\|\widehat{w}_{x}(t, .)\right\|_{L^{2}}^{2}+\left\|\widehat{w}_{t}(t, .)\right\|_{L^{2}}^{2}\right),
$$

where $a_{3}=\max \left\{a_{0}\left(1+e_{\infty}\right)+\pi_{\infty}\left(1+a_{0}\right)+h_{\infty}, f_{\infty}\right\}>0, a_{0}>0$.

Besides, as

$$
f(x, 0)=0, h(x, 0)=\pi(x), f_{\xi}(x, 0)=0, f_{x}(x, x)=0
$$

we get,

$$
\left\|\widehat{v}_{t}(t, .)\right\|_{L^{2}}^{2} \leq a_{4}\left(\left\|\widehat{w}_{t}(t, .)\right\|_{L^{2}}^{2}+\left\|\widehat{w}_{x}(t, .)\right\|_{L^{2}}^{2}\right),
$$

where $a_{4}=\max \left\{1+e_{\infty}+h_{\infty}+\left(h_{\xi}\right)_{\infty}+\iota f_{\infty}, f_{\infty}+a_{0}\left(f_{\xi \xi}\right)_{\infty}\right\} \geq 0$.

Also, as $\widehat{w}(t, 0)=\widehat{w}(t, x)-\int_{0}^{x} \widehat{w}_{y}(t, y) d y$ we find

$$
\left\|\widehat{v}_{x}(t, .)\right\|_{L^{2}}^{2} \leq a_{5}\left(\left\|\widehat{w}_{x}(t, .)\right\|_{L^{2}}^{2}+\left\|\widehat{w}_{t}(t, .)\right\|_{L^{2}}^{2}\right)
$$

where $a_{5}=\max \left\{1+a_{0} e_{\infty}+a_{0}\left(e_{x}\right)_{\infty}+h_{\infty}+\left(h_{x}\right)_{\infty}+\pi_{\infty}^{\prime}\left(1+a_{0}\right), f_{\infty}+\left(f_{x}\right)_{\infty}\right\}$.

Finally, we have $\left|\widehat{v}_{t}(t, 0)\right|^{2} \leq 4\left|\widehat{w}_{t}(t, 0)\right|^{2}$. Accordingly, there exits $\zeta_{2}>0$ such that $\widehat{\Pi}(t) \leq \zeta_{2} \widehat{\Xi}(t)$.

This implies that the system (12)-(14) is exponentially stable in the sense of the $\widehat{\Xi}$ norm.

Remark 1. The proof of Theorem 2 is performed in three steps: first, the stability of the observer target system; second, the mapping between the observer plant system and the observer target, and the computation of the observer based control law; finally, the stability of the observer plant system.

\section{Output injection gain}

The first goal of this section is to prove the existence and uniqueness solution using Lumer-Phillips's theorem. The second one is the stability study of the estimation error system.

\subsection{Well-posedness problem}

In the next, we use semigroup theory (Further discussion in this theory in [19]) to prove the existence and uniqueness of the proposed observer solutions. Then, by proving the existence and uniqueness of the estimation error model, we conclude the existence and uniqueness of the proposed observer system. In addition, due to the presence of a nonlinear and complex relation resulting from the bit-rock interaction at the tip boundary, the well-posedness of the estimation error system becomes not trivial. Hence, in the next, we treat this contribution using the semi-group theory.

We denote the estimation error by $\tilde{v}=v-\widehat{v}$. Let $T>0$, the natural solution of the Cauchy problem is written in this form

$$
\begin{aligned}
& \tilde{v}_{t t}(t, x)=\tilde{v}_{x x}(t, x)-l \tilde{v}_{t}(t, x) \\
& \tilde{v}_{t}(t, 1)=-\frac{\sqrt{I G J}}{c_{a}} \tilde{v}_{x}(t, 1)-\gamma \tilde{v}(t, 1) \\
& \tilde{v}_{t t}(t, 0)=a \tilde{v}_{x}(t, 0)+a F\left(\tilde{v}_{t}(t, 0)\right) \\
& \tilde{v}(0, x)=\tilde{v}^{0}(x), \quad \tilde{v}_{t}(0, x)=\tilde{v}^{1}(x)
\end{aligned}
$$

where $x \in(0,1), t \in(0, T), \tilde{v}^{0} \in K:=\left\{\tilde{v} \in H^{1}(0,1) ; \tilde{v}^{0}(0)=0\right\}$, and $\tilde{v}^{1} \in L^{2}(0,1)$. 
The vector space $K$ is equipped with the scalar product

$$
\left\langle\tilde{v}^{1}(t, x), \tilde{v}^{2}(t, x)\right\rangle_{K}=\int_{0}^{1} \tilde{v}_{x}^{1}(t, x) \tilde{v}_{x}^{2}(t, x) d x .
$$

It is obvious that $K$ is a Hilbert space.

Let us introduce $Y=\left(\tilde{v}(t, x), \tilde{v}_{t}(t, x), \tilde{v}(t, 1), \tilde{v}_{t}(t, 0)\right)^{T}$. Eqs. (29)-(32) can be compactly written as

$$
\left\{\begin{array}{l}
\dot{Y}(t)=A Y(t)+H(Y(t))+f(t) \\
Y(0)=Y_{0}
\end{array}\right.
$$

where

$$
A=\left(\begin{array}{cccc}
0 & 1 & 0 & 0 \\
\partial_{x x} & -\iota & 0 & 0 \\
\frac{\sqrt{I G J}}{C_{a}}\left\langle\delta_{1}^{\prime}(x), .\right\rangle-\gamma & 0 & 0 & 0 \\
-a\left\langle\delta_{0}^{\prime}(x), .\right\rangle & 0 & 0 & 0
\end{array}\right), H(Y(t))=\left(\begin{array}{c}
0 \\
0 \\
0 \\
a F\left(\tilde{v}_{t}(t, 0)\right)
\end{array}\right) \text {, and } f(t)=\left(\begin{array}{c}
0 \\
0 \\
L \sqrt{\frac{I}{G J}} \omega(t) \\
0
\end{array}\right) \text { such as } \delta \text { denotes the }
$$

Dirac function for which $\left\langle\delta_{1}^{\prime}(x), \tilde{v}(t, x)\right\rangle=-\tilde{v}_{x}(t, 1)$ and $\left\langle\delta_{0}^{\prime}(x), \tilde{v}(t, x)\right\rangle=-\tilde{v}_{x}(t, 0)$.

Firstly, let us consider the problem (33) with $H(Y)=0$ and $f(t)=0$, consequently we have the next Theorem.

Theorem 3. The operator $A$ generates a $C_{0}$ semigroup $S(t), t \geq 0$ of contractions on $E$.

Proof. Let us consider the following space

$$
E=\left\{\left(\begin{array}{c}
\tilde{v}(t, x) \\
\tilde{v}_{t}(t, x) \\
\tilde{v}(t, 1) \\
\tilde{v}_{t}(t, 0)
\end{array}\right), \tilde{v} \in K, \tilde{v}_{t} \in L^{2}([0,1]), \tilde{v}(t, 1) \in \mathbb{R}, \tilde{v}_{t}(t, 0) \in \mathbb{R}\right\}
$$

This vector space $E$ is equipped with theinner-product

$$
\begin{aligned}
\left\langle\left(\begin{array}{c}
\tilde{v}^{1}(t, x) \\
\tilde{v}_{t}^{1}(t, x) \\
\tilde{v}^{1}(t, 1) \\
\tilde{v}_{t}^{1}(t, 0)
\end{array}\right),\left(\begin{array}{c}
\tilde{v}^{2}(t, x) \\
\tilde{v}_{t}^{2}(t, x) \\
\tilde{v}^{2}(t, 1) \\
\tilde{v}_{t}^{2}(t, 0)
\end{array}\right)\right\rangle_{E} & =\left\langle\tilde{v}^{1}, \tilde{v}^{2}\right\rangle_{K}+\left\langle\tilde{v}_{t}^{1}, \tilde{v}_{t}^{2}\right\rangle_{L^{2}[0,1]}+\left\langle\tilde{v}^{1}(t, 1), \tilde{v}^{2}(t, 1)\right\rangle_{\mathbb{R}} \\
& +\left\langle\tilde{v}_{t}^{1}(t, 0), \tilde{v}_{t}^{2}(t, 0)\right\rangle_{\mathbb{R}} .
\end{aligned}
$$

We denote by $\|\cdot\|$ the norm in $E$ associated to this scalar product.

Let $A: D(A) \subset E \rightarrow E$ be the linear operator defined by

$D(A)=\left\{\left(\begin{array}{c}\tilde{v}_{(t, x)} \\ \tilde{v}_{t}(t, x) \\ \tilde{v}(t, 1) \\ \tilde{v}_{t}(t, 0)\end{array}\right) \in E, \tilde{v} \in H^{2}(0,1), \tilde{v}_{t} \in K, \tilde{v}_{x}(t, 1)=\tilde{v}_{x}(t, 0)=0, \tilde{v}(t, 1) \in \mathbb{R}, \tilde{v}_{t}(t, 0) \in \mathbb{R}\right\}$.

We have

$$
A\left(\begin{array}{c}
\tilde{v}(t, x) \\
\tilde{v}_{t}(t, x) \\
\tilde{v}(t, 1) \\
\tilde{v}_{t}(t, 0)
\end{array}\right)=\left(\begin{array}{c}
\tilde{v}_{t}(t, x) \\
\tilde{v}_{x x}(t, x)-\iota \tilde{v}_{t}(t, x) \\
-\frac{\sqrt{I G}}{c_{a}} \tilde{v}_{x}(t, 1)-\gamma \tilde{v}(t, 1) \\
a \tilde{v}_{x}(t, 0)
\end{array}\right)
$$

Moreover

$$
\langle A Y, Y\rangle_{E}=-\iota \int_{0}^{1} \tilde{v}_{t}^{2} d x-\gamma \tilde{v}(t, 1)^{2} \leq 0, \forall Y \in D(A) .
$$

It is easy to verify that $\forall y=\left(\begin{array}{l}f_{1} \\ f_{2} \\ f_{3} \\ f_{4}\end{array}\right) \in E$, there exists $w=\left(\begin{array}{l}w_{1} \\ w_{2} \\ w_{3} \\ w_{4}\end{array}\right) \in D(A)$ such that $w-A w=y$. Then, $D(A)$ is dense in $E$ and $A$ is closed. Hence, using the Lumer-Phillips theorem (Theorem A.4 in [20]) $A$ is the infinitesimal generator of a strongly continuous group of isometries $S(t), t \geq 0$, on $E$.

Now, we are going to prove the existence and uniqueness of the system (33) with $H(Y)$ and $f(t)$ are different from zero.

Theorem 4. Let $f \in L^{1}([0, T], E)$ and $Y_{0} \in D(A)$, then the problem $\dot{Y}(t)=A Y(t)+H(Y)+f(t)$ has a unique solution

$$
Y \in C^{1}([0, T], E) \bigcap C^{0}([0, T], D(A))
$$

given by:

$$
Y(t)=S(t) Y(0)+\int_{0}^{t} S(t-s)(H(Y(s))+f(s)) d s
$$


To prove the Theorem 4, we need the next lemmas:

Lemma 2. The nonlinear operator $H(Y)$ is dissipative and locally Lipschitz.

Proof. Recall that the nonlinear function $F$ due to the bit-rock contact is given by

$$
\begin{aligned}
F\left(\tilde{v}_{t}(t, 0)\right) & =-\frac{L}{G J} T\left(\frac{1}{L} \sqrt{\frac{G J}{I}} \tilde{v}_{t}(t, 0)\right), \\
& =-c_{b} \sqrt{\frac{1}{G J I}} \tilde{v}_{t}(t, 0)-\frac{L}{G J} W_{o b} R_{b} \mu\left(\frac{1}{L} \sqrt{\frac{G J}{I}} \tilde{v}_{t}(t, 0)\right) \operatorname{sgn}\left(\frac{1}{L} \sqrt{\frac{G J}{I}} \partial_{t} \tilde{v}_{t}(t, 0)\right)
\end{aligned}
$$

where $\mu$ is as [6]

$$
\mu\left(\frac{1}{L} \sqrt{\frac{G J}{I}} \tilde{v}_{t}(t, 0)\right)=\mu_{c b}+\left(\mu_{s b}-\mu_{c b}\right) e^{-\frac{\gamma_{b}}{v_{f}}\left|\frac{1}{L} \sqrt{\frac{G}{I}} \tilde{v}_{t}(t, 0)\right|} .
$$

After computing

$$
\begin{aligned}
\langle H(Y(t))), Y(t)\rangle_{E}= & F\left(\tilde{v}_{t}(t, 0)\right) \tilde{v}_{t}(t, 0) \\
= & -c_{b} \sqrt{\frac{1}{G J I}}\left(\partial_{t} \tilde{v}(t, 0)\right)^{2}-\frac{L}{G J} W_{o b} R_{b}\left(\mu_{c b}+\left(\mu_{s b}-\mu_{c b}\right) e^{-\frac{\gamma_{b}}{v_{f}}\left|\frac{1}{L} \sqrt{\frac{G l}{T}} \tilde{v}_{t}(t, 0)\right|}\right) \\
& \times \operatorname{sgn}\left(\frac{1}{L} \sqrt{\frac{G J}{I}} \tilde{v}_{t}(t, 0)\right) \tilde{v}_{t}(t, 0) .
\end{aligned}
$$

Consequently, $\langle H(Y(t)), Y(t)\rangle_{E} \leq 0$ as $\mu_{s b} \geq \mu_{c b}$. This implies that the operator $H(Y)$ is dissipative.

It's easy to verify that, $H$ is locally Lipschitz. Accordingly, the operator $H(Y)$ is dissipative and locally Lipschitz.

Lemma 3. For any function $f \in L^{1}([0, T], E)$, and any initial condition $Y_{0} \in D(A)$, the problem (33) has at most one solution in $C^{1}([0, T], E) \cap C^{0}([0, T], D(A))$.

Proof. Suppose $Y_{1}$ and $Y_{2}$ are two solutions of (33) in the class $C^{1}([0, T], E) \cap C^{0}([0, T], D(A))$. Then the difference $Y=$ $Y_{1}-Y_{2}$ is an element of $C^{1}([0, T], E) \cap C^{0}([0, T], D(A))$ which satisfies the next system

$$
\left\{\begin{array}{l}
\dot{Y}(t)=A Y(t)+H(Y(t)) \\
Y(0)=0
\end{array} .\right.
$$

Since $A$ and $H(Y)$ are dissipative, we get

$$
\langle\dot{Y}(t), Y(t)\rangle_{E}=\langle A Y(t), Y(t)\rangle_{E}+\langle H(Y(t)), Y(t)\rangle_{E} \leq 0 .
$$

Then

$$
\frac{1}{2} \frac{d}{d t}\|Y(t)\|_{E} \leq 0 \Rightarrow\|Y(t)\|_{E}=0 .
$$

Hence, the problem (34) has a unique solution $Y(t)=0$ for every $Y_{0} \in D(A)$, thus proves that $Y_{1}=Y_{2}$ and shows that (33) has a solution in $C^{1}([0, T], E) \cap C^{0}([0, T], D(A))$, then this one is unique.

Proof. (of Theorem 4) By applying the two Lemmas given above and from results given in (Theorem 4.2 in [21], [19,20,22]), it is easy to prove that our system (33) has a unique solution.

\subsection{Stability analysis of estimation error system}

Here, the main contribution is the stabilization of the estimation error system and the computing of the output injection gain $\gamma$. Let us recall the observer based control system

$$
\begin{aligned}
\widehat{v}_{t t}(t, x) & =\widehat{v}_{x x}(t, x)-\imath \widehat{v}_{t}(t, x) \\
\widehat{v}_{x}(t, 1) & =\Omega(t)-\gamma(v(t, 1)-\widehat{v}(t, 1)) \\
\widehat{v}_{t t}(t, 0) & =a \widehat{v}_{x}(t, 0)+a b \widehat{v}_{t}(t, 0) .
\end{aligned}
$$

In order to fructify the control law (27), it is important to identify the output injection gain $\gamma$. The output injection gain $\gamma$ should be designed using the backstepping procedure (more details in [13]). Following the above mentioned method, the output injection is applied in the boundary as well as in the whole spacial domain $(\forall x \in[0 ; 1])$. The analysis takes into account the estimated error $\widetilde{v}=v-\widehat{v}$ is as

$$
\tilde{v}_{t t}(t, x)=\widetilde{v}_{x x}(t, x)-\iota \widetilde{v}_{t}(t, x)
$$




$$
\begin{aligned}
& \tilde{v}_{x}(t, 1)=-\gamma \widetilde{v}(t, 1) \\
& \widetilde{v}_{t t}(t, 0)=a \widetilde{v}_{x}(t, 0)+a b \widetilde{v}_{t}(t, 0)
\end{aligned}
$$

In order to get the output injection gain $\gamma$ that ensure that the estimation error system go to zero, we introduce a backstepping transformation to convert the error system into the next error target system (i.e. $\widetilde{v}(t, x) \rightarrow \widetilde{w}(t, x)$ )

$$
\begin{aligned}
& \widetilde{w}_{t t}(t, x)=\widetilde{w}_{x x}(t, x)-\imath \widetilde{w}_{t}(t, x) \\
& \widetilde{w}_{x}(t, 1)=0 \\
& \widetilde{w}_{t t}(t, 0)=a e^{-\eta} \widetilde{w}_{x}(t, 0)-(2 a \epsilon+1) \widetilde{w}_{t}(t, 0) .
\end{aligned}
$$

We propose the following backstepping transformation

$$
\begin{aligned}
\widetilde{v}(t, x)= & \widetilde{w}(t, x)-\int_{x}^{1} A(x, \xi) \widetilde{w}(t, \xi) d \xi-\int_{x}^{1} B(x, \xi) \widetilde{w}_{t}(t, \xi) d \xi \\
& -\int_{x}^{1} C(x, \xi) \widetilde{w}_{\xi}(t, \xi) d \xi
\end{aligned}
$$

Lemma 4. Let us introduce the function

$$
V(t)=\frac{1}{2}\left[\int_{0}^{1}\left(e^{-\eta}\left(\tilde{w}_{x}\right)^{2}+e^{-\eta}\left(\tilde{w}_{t}\right)^{2}+\epsilon e^{-\eta x}(1-x) \tilde{w}_{t} \tilde{w}_{x}\right) d x+\frac{1}{a}\left(\tilde{w}_{t}(t, 0)\right)^{2}\right]
$$

with $\frac{1}{2}>\epsilon>0, \eta \leq-\frac{2+\epsilon}{\epsilon(1-x)}$, in which $x \in[0,1[$, and the $\tilde{\Xi}$ norm where

$$
\tilde{\Xi}^{2}(t)=\left\|\widetilde{w}_{t}\right\|_{L^{2}([0,1])}^{2}+\left\|\widetilde{w}_{x}\right\|_{L^{2}([0,1])}^{2}+\left|\tilde{w}_{t}(t, 0)\right|^{2} .
$$

Then

$$
n_{1} \tilde{\Xi}^{2}(t) \leq V(t) \leq n_{2} \tilde{\Xi}^{2}(t)
$$

where $n_{1}=\min \left\{\frac{e^{-\eta}}{2}-\frac{\epsilon e^{-\eta}}{4}, \frac{1}{2 a}\right\}$ and $n_{2}=\max \left\{\frac{e^{-\eta}}{2}+\frac{\epsilon e^{-\eta}}{4}, \frac{1}{2 a}\right\}$

Proof. The proof is similar to the one of Lemma 1.

Our first result, in this section, on stabilization is given by the following Theorem.

Theorem 5. (Estimation error target system stability) Consider system (38)-(40), with initial condition $\tilde{w}_{0}=\tilde{w}(0, x) \in L^{2}([0,1])$. Then the zero equilibrium of (38)-(40) is exponentially stable in the sense of the next norm

$$
\tilde{\Xi}^{2}(t)=\left\|\widetilde{w}_{t}\right\|_{L^{2}([0,1])}^{2}+\left\|\widetilde{w}_{x}\right\|_{L^{2}([0,1])}^{2}+\left|\tilde{w}_{t}(t, 0)\right|^{2} .
$$

Proof. The proof is similar to the one of Theorem 1.

To get the output injection gain $\gamma$ that guarantee that the estimation error decays to zero, we use a backstepping transformation. Then, plugging (41) into (35)-(37), we obtain the next kernel PDE

$$
\begin{aligned}
& C_{x x}(x, \xi)=C_{\xi \xi}(x, \xi) \\
& A_{x x}(x, \xi)=A_{\xi \xi}(x, \xi) \\
& B_{x x}(x, \xi)=B_{\xi \xi}(x, \xi)
\end{aligned}
$$

with the next boundary conditions

$$
\begin{aligned}
C_{x}(x, x)= & 0, \quad A_{x}(x, x)=0, \quad B_{x}(x, x)=0 \\
A_{\xi}(x, 1)= & B_{\xi}(x, 1)=C(x, 1)=0, \\
B(1,1)= & B(0,0)=A(0,0)=0, \\
A_{\xi \xi}(0, \xi)= & (\iota+a b) B_{\xi \xi}(0, \xi)+a A_{x}(0, \xi) \\
C_{\xi \xi}(0, \xi)= & a C_{x}(0, \xi), \quad C(0,0)=-1 \\
B_{\xi \xi}(0, \xi)= & (\iota+a b) A(0, \xi)-(\iota+a b) C_{\xi}(0, \xi) \\
& +a B_{x}(0, \xi)-\left(\iota^{2}+\iota a b\right) B(0, \xi) .
\end{aligned}
$$




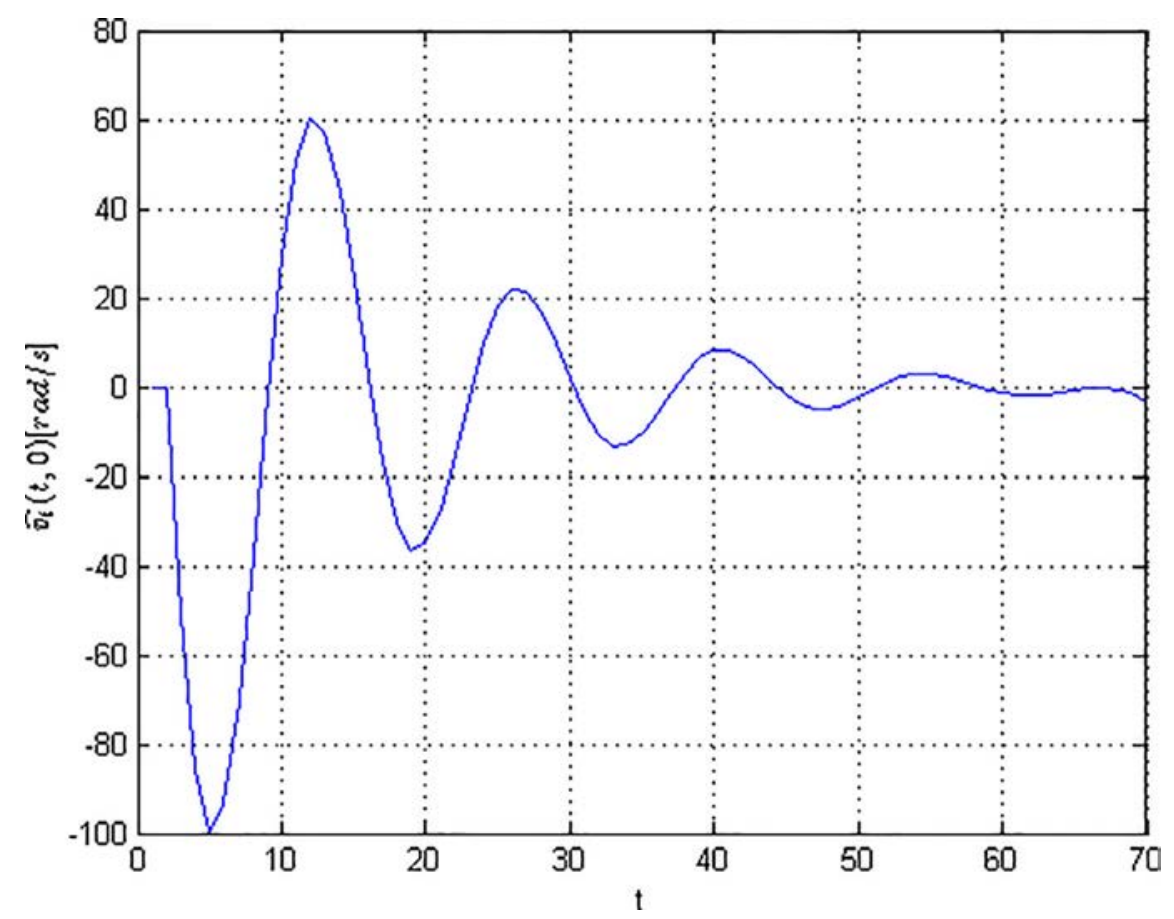

Fig. 2. Stabilization at the bottom extremity of $\widehat{v}_{t}(t, 0)$.

Plugging (41) into (36), we find the output injection gain $\gamma=A(1,1)$. It is easy to verify that $C(x, \xi), A(x, \xi)$ and $B(x, \xi)$ satisfies a wave PDE which the general solution is given by $F(x, \xi)=\Psi(x-\xi)+\Phi(x-\xi)$. Then, this equations are defined on a triangular domain $\Delta=\left\{(x, \xi) \in \mathbb{R}^{2}: 0 \leq \xi \leq x \leq 1\right\}$.

The backstepping transformation should be invertible. Hence, to convert (35)-(37) into (38)-(40) (i.e. $\tilde{w}(t, x) \rightarrow \widetilde{v}(t, x))$, we introduce the next inverse backstepping transformation:

$$
\begin{aligned}
\widetilde{w}(t, x)= & \widetilde{v}(t, x)+\int_{x}^{1} M(x, \xi) \widetilde{v}(t, \xi) d \xi+\int_{x}^{1} N(x, \xi) \widetilde{v}_{t}(t, \xi) d \xi \\
& +\int_{x}^{1} J(x, \xi) \widetilde{v}_{\xi}(t, \xi) d \xi .
\end{aligned}
$$

Plugging (42) into (38)-(40), we obtain the next kernel PDE

$$
\begin{aligned}
M_{\xi \xi}(x, \xi) & =M_{x x}(x, \xi) \\
N_{\xi \xi}(x, \xi) & =N_{x x}(x, \xi) \\
J_{\xi \xi}(x, \xi) & =J_{x x}(x, \xi)
\end{aligned}
$$

with the next boundary conditions

$$
\begin{aligned}
0= & M_{x}(x, x), \quad J_{x}(x, x)=0, \quad N_{x}(x, x)=0 \\
0= & M(x, 1)-J_{\xi}(x, 1), \quad 0=N(x, 1)=J(x, 1) \\
0= & M_{\xi}(x, 1), \quad N_{\xi}(x, 1)=0 \\
1= & J(0,0), M(0,0)=0, N(0,0)=0 \\
0= & (2 a \epsilon+1-\iota) N_{\xi \xi}(0, \xi)+M_{\xi \xi}(0, \xi)-a e^{-\eta} M_{x}(0, \xi) \\
0= & -a e^{-\eta} N_{x}(0, \xi)+(\iota-(2 a \epsilon+1)) J_{\xi}(0, \xi)+N_{\xi \xi}(0, \xi)+(2 a \epsilon+1-\iota) M(0, \xi) \\
& +\left(\iota^{2}-(2 a \epsilon+1) \iota\right) N(0, \xi) \\
0= & -a e^{-\eta} J_{x}(0, \xi)+J_{\xi \xi}(0, \xi) .
\end{aligned}
$$

It is easy to find the existence and uniqueness of the solution of the kernel PDE. Then, this equations are defined on a triangular domain $\Delta=\left\{(x, \xi) \in \mathbb{R}^{2}: 0 \leq \xi \leq x \leq 1\right\}$.

The main result regarding the estimation error system stability is summarized in the next Theorem. 


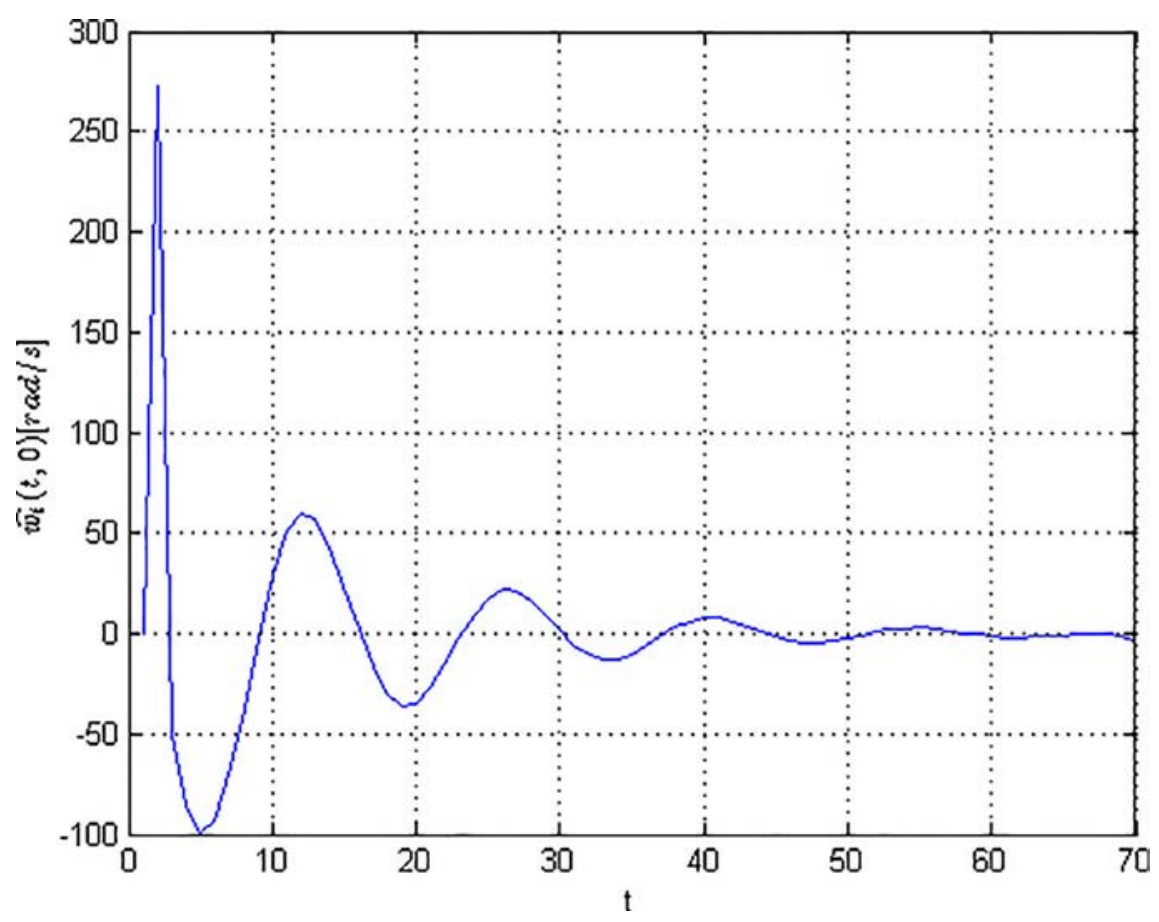

Fig. 3. Stabilization at the bottom extremity of $\widehat{w}_{t}(t, 0)$.

Theorem 6. (Plant estimation error system stability) Consider system (35)-(37) with the output injection gain given by $\gamma=$ $A(1,1)$. Then the equilibrium $\tilde{v}=0$ is exponentially stable in the sense of the next norm

$$
\tilde{\Pi}^{2}(t)=\|\tilde{v}(t, .)\|_{L^{2}([0,1])}^{2}+\left\|\tilde{v}_{t}(t, .)\right\|_{L^{2}([0,1])}^{2}+\left\|\tilde{v}_{x}(t, .)\right\|_{L^{2}([0,1])}^{2}+\left|\tilde{v}_{t}(t, 0)\right|^{2} .
$$

The strategy for the proof of Theorem 6 is the same as in the previous section.

Proof. We will prove that there exist $\alpha_{1}>0$ and $\alpha_{2}>0$ such that $\alpha_{1} \tilde{\Xi}(t) \leq \tilde{\Pi}(t) \leq \alpha_{2} \tilde{\Xi}(t)$. The proof of this theorem is similar to the observer design case (Theorem 2).

By combining the observer based control law and the estimation error, we find the next feedback control law

$$
\begin{aligned}
\Omega(t)= & \frac{1}{1-l(1,1)}\left[k(1,1) \widehat{v}(t, 1)+\int_{0}^{1} k_{x}(1, \xi) \widehat{v}(t, \xi) d \xi+p(1,1) \widehat{v}_{t}(t, 1)\right. \\
& \left.+\int_{0}^{1} p_{x}(1, \xi) \widehat{v}_{t}(t, \xi) d \xi+\int_{0}^{1} l_{x}(1, \xi) \widehat{v}_{\xi}(t, \xi) d \xi+\beta^{\prime}(1) \widehat{v}(t, 0)\right] \\
& +t A(1,1)(v(t, 1)-\widehat{v}(t, 1)) .
\end{aligned}
$$

\section{Numerical simulation}

We proceed to test the effectiveness of the boundary observer based control law leading to an exponential stability result of the torsional vibration. Our numerical simulations take in consideration the next physical parameters:

The numerical values corresponding to the dynamic of the torsional variable were taken from the work of [4,18]. In practice to optimize drilling operations, the driller operator typically controls the drilling parameters at the surface, such as the drill string rotational speed, the weight on the bit, and the drilling fluid viscosity. Here, we are limited to the vibration effect and its stability impact for the observer based control system. As expected by Theorems 1 and 2, from (Figs. 2 and 3 ), the constructed observers from the target system and the plant tend to zero (exponentially) as time goes to infinity (Table 1).

The both Figs. 2 and 3 show the behavior of the velocity in the bottom extremity of the observer plant and target systems, respectively.

Figs. 2 and 3 concern the observer-based control case (i.e. system (12-14) with the control law (43), solved in Theorems 1 and 2 . 
Table 1

Different physical parameters.

\begin{tabular}{lll}
\hline Variable & Value & Description \\
\hline$L$ & $2000 \mathrm{~m}$ & Drillstring length \\
$I$ & $0.095 \mathrm{~kg} \cdot \mathrm{m}$ & Inertia per unit length \\
$I_{b}$ & $311 \mathrm{~kg} \cdot \mathrm{m}^{2}$ & Inertia at the drillstring bottom \\
$J$ & $1.19 .10^{5} \mathrm{~m}^{4}$ & Geometrical moment of inertia \\
$c_{a}$ & $2000 \mathrm{Nm}_{\text {. }} \mathrm{rad}^{-1}$ & Sliding torque coefficient \\
$G$ & $79.310^{9} \mathrm{~N} \mathrm{~m}^{-2}$ & Shear modulus \\
\hline
\end{tabular}

\section{Conclusion}

In this paper, an observer based control law has been designed taking into account in domain damping for a wave PDE. We introduce and solve stabilization problems for boundary observer system. The stability of the closed-loop system is proved using a Lyapunov functional and the backstepping techniques. Also, an output injection gain parameter was defined leading to a $L^{2}$ exponential stability of the estimation error system. Further analysis is required to quantify the impact of other modeling errors. Other direction of future work aim at finding other drilling stability factors like the drill string and fluid interaction.

\section{References}

[1] J.D. Jansen, Nonlinear Dynamics of Oilwell Drill Strings, Delft University of Technology, 1993, doi:10.1007/s10596-016-9576-1.44.

[2] S. Toumi, L. Beji, R. Mlayeh, A. Abichou, Stability analysis of coupled torsional vibration and pressure in oilwell drillstring system, Int. J. Control 90 (3) (2017) $10.1080 / 00207179.2016 .1278269$

[3] P. Rouchon, Flatness and Stick-Slip Stabilization, Ecole des Mines de Paris, Technical Report 492, France, 1998. 10.1.1.648.8232.

[4] B. Saldivar, I. Boussaada, H. Mounier, S. Niculescu, Analysis and control of oilwell drilling vibrations, a time-delay systems approach, Advances in Industrial Control, Springer, New York, 2015, doi:10.1007/978-3-319-15747-4.

[5] J.J. Bailey, I. Finnie, An analytical study of drill-string vibration, J. Eng. Ind. 82 (2) (1960) 122-127, doi:10.1115/1.3663017

[6] B. Saldivar, S. Mondié, J.J. Loiseau, V. Rasvan, Stick-slip oscillations in oillwell drill strings: distributed parameter and neutral type retarded model approaches, IFAC Proc. Vol. 44 (1) (2011) 284-289, doi:10.3182/20110828-6-IT-1002.00084.

[7] S. Toumi, L. Beji, R. Mlayeh, A. Abichou, Boundary observer design for hyperbolic PDE in rotary drilling systems, in: Proceedings of the IEEE Fifty-Fifth Conference on Decision and Control (CDC), Las Vegas, USA, 2016, pp. 2128-2133, doi:10.1109/CDC.2016.7798578.

[8] X. Liu, N. Vlajic, X. Long, G. Meng, Nonlinear oscillations of a flexible rotor with a drill bit: stick-slip and delay effects, Nonlinear Dyn. 72 (1-2) (2013) 61-77, doi: $10.1007 / \mathrm{s} 11071-012-0690-\mathrm{X}$

[9] C. Roman, D. Bresch-Pietri, E. Cerpa, C. Prieur, O. Sename, Backstepping observer based-control for an anti-damped boundary wave PDE in presence of in-domain viscous damping, Proceedings of the IEEE Fifty-Fifth Conference on Decision and Control (CDC) 50 (2016), doi:10.1109/CDC.2016.7798326.

[10] S. Toumi, L. Beji, R. Mlayeh, A. Abichou, Exponential stabilization of torsional vibrations in rotary drilling systems, IFAC-PapersOnLine 50 (1) (2017) 1565-1570, doi:10.1016/j.ifacol.2017.08.310.

[11] S. Toumi, R. Mlayeh, L. Beji, A. Abichou, Stability analysis of oilwell drilling torsional vibrations, in: Proceedings of the Twenty-Fourth Mediterranean Conference on Control and Automation (MED), (2016) 677-682, doi:10.1109/MED.2016.7535945.

[12] D. Bresch-Pietri, M. Krstic, Output-feedback adaptive control of a wave PDE with boundary antidamping, Automatica 50 (2014) 1407-1415, doi:10.1016/ j.automatica.2014.02.040.

[13] M. Krstic, A. Smyshlyaev, Boundary Control of PDEs: A Course on Backstepping Designs, 16, SIAM, 2008. ISBN: 9780898716504.

[14] R. Vazquez, M. Krstic, J. Coron, G. Bastin, Local exponential $h^{2}$ stabilization of a $2 \times 2$ quasilinear hyperbolic system using backstepping, in: Proceedings of the Fiftieth Conference on Decision Control, Orlando, FL, USA, 2011, pp. 1329-1334, doi:10.1109/CDC.2011.6161075.

[15] P.V. Kokotovic, The joy of feedback: nonlinear and adaptive, control systems, Mag. IEEE 12 (1992) 07-17, doi:10.1109/37.165507.

[16] A. Kyllingstad, E.M. Halsey, A study of slip/stick motion of the bit 1988, 369-373, 10.2118/16659-PA.

[17] S. Toumi, L. Beji, R. Mlayeh, A. Abichou, Stabilization of stick-slip oscillations integrating fluid injection in oilwell drillstring system, Proceedings of the European Control Conference (2016) 352-357, doi:10.1109/ECC.2016.7810310.

[18] E. Navarro, D. Corts, Sliding-mode of a multi-dof oilwell drillstring with stick-slip oscillations, in: Proceedings of the American Control Conference, New York City, USA, 2007, pp. 3837-3842, doi:10.1109/ACC.2007.4282198.

[19] A. Pazy, Semigroups of linear operators and applications to partial differential equations, 44, Springer, New York, 1983, doi:10.1007/ 978-1-4612-5561-1.

[20] J.M. Coron, Control and Nonlinearity, 136, American Mathematical Society, 20071098765432112111009.

[21] M. Slemrod, Feedback stabilization of a linear control system in hilbert space with ana priori bounded control, Math. Control Signals Syst. 2 (3) (1989) 265-285, doi:10.1007/BF02551387.

[22] T.D. Nguyen, O. Egeland, Stabilization of towed cables, Proceedings of the Forty-Third IEEE Conference on Decision and Control, CDC. 5 (2004) 50595064, doi:10.1109/CDC.2004.1429609. 